Meta

Journal des traducteurs

Translators' Journal

\title{
Les pulsations de la traduction
}

\section{Walter Moser}

Volume 30, numéro 1, mars 1985

Interprétation de conférence

URI : https://id.erudit.org/iderudit/003672ar

DOI : https://doi.org/10.7202/003672ar

Aller au sommaire du numéro

Éditeur(s)

Les Presses de l'Université de Montréal

ISSN

0026-0452 (imprimé)

1492-1421 (numérique)

Découvrir la revue

Citer cet article

Moser, W. (1985). Les pulsations de la traduction. Meta, 30(1), 7-18.

https://doi.org/10.7202/003672ar

Ce document est protégé par la loi sur le droit d'auteur. L'utilisation des services d'Érudit (y compris la reproduction) est assujettie à sa politique d'utilisation que vous pouvez consulter en ligne.

https://apropos.erudit.org/fr/usagers/politique-dutilisation/
Cet article est diffusé et préservé par Érudit.

Érudit est un consortium interuniversitaire sans but lucratif composé de l’Université de Montréal, l'Université Laval et l'Université du Québec à Montréal. Il a pour mission la promotion et la valorisation de la recherche. https://www.erudit.org/fr/ 


\title{
LES PULSATIONS DE LA TRADUCTION
}

\author{
WALTER MOSER
}

Sous ce titre quelque peu énigmatique, je ne développerai pas de nouvelle théorie ni ne construirai de nouveau modèle de la traduction. J'établirai encore moins de règles pratiques pour l'opération traduisante. Je ne profiterai même pas de la possibilité d'extraire " pulsions " de " pulsations " afin de traiter des liens entre psychanalyse et traduction.

Je propose plutôt de relire quelques textes bien connus sur la traduction, de faire le parcours de quelques lieux communs dans notre discours sur la traduction. Notre fréquentation de ces lieux leur donne un aspect si familier que leur évidence pourrait les avoir rendus invisibles. Nous les pratiquons, mais nous ne les voyons peut-être plus. Voilà pourquoi j'aimerais en faire apparaître quelques-uns dans cette étude et inviter le lecteur à interroger avec moi leurs présuppositions.

Pour ce faire je m'inspirerai du projet qui avait animé Jacques Derrida quand il a fait ressortir les contours du phonocentrisme dans la linguistique de F. de Saussure :

Nous voudrions (plutôt) annoncer les limites et les présuppositions de ce qui semble ici aller de soi et garder pour nous les caractères et la validité de l'évidence. (1967: 58)

Plus que jamais, la traduction est devenue de nos jours un des termes clés sur lesquels convergent les intérêts des sémioticiens, des philosophes, des poétologues, bref de tous ceux qui font de la pratique des signes leur objet d'analyse et de réflexion. Dans leurs textes, les termes " traduire ", " traduction ", " opération traduisante " se trouvent être soumis à des changements de signification d'une amplitude extrêmement grande : voilà pourquoi je parlerai des pulsations sémantiques et opératoires de la notion qui constitue la raison d'être même de la revue Meta.

Certes, la traduction n'est pas le seul terme, aujourd'hui, à subir des pulsations d'une telle amplitude. On pourrait établir toute une série de termes qui suivent la traduction, surtout dans son mouvement d'expansion et de généralisation : texte, intertexte, discours feraient certainement partie de cette série. Cependant, parmi ces termes à la mode, la traduction mérite une attention particulière de notre part. C'est que ce terme n'exécute pas seulement une pulsation quant à l'étendue quantitative de son terrain sémantique, mais aussi une réinterprétation qualitative ${ }^{1}$. Celle-ci se joue dans la bipolarité, dans l'ambivalence qui est constitutive de l'opération traduisante : elle comporte un élément stable (repérable dans les notions de fidélité, d'équivalence, d'identité du sens, dans le maintien du message, etc.) et un élément mobile et risqué (exprimé par le préfixe trans- qui contient l'idée de transfert et de déplacement nécessairement impliquée dans l'opération traduisante). Aujourd'hui, l'intérêt des écrits sur la traduction porte nettement sur sa mobilité ainsi que sur son potentiel d'altération productive et les valorise unilatéralement. La traduction est vue et présentée en premier lieu comme une opératrice de changement, de transformation, de production sémiotique et moins comme une stabilisatrice du sens et des échanges sémiotiques . 
À titre d'hypothèse on pourrait en conclure que notre intérêt accru et spécifique pour la traduction doit être à la fois indicateur et opérateur d'un bouleversement majeur de la pratique des signes dans notre société. On pourrait parler d'une crise généralisée, ou d'une catastrophe, au sens donné à ce mot par René Thom.

Vérifier cette hypothèse serait une tâche démesurée dans les limites de cet article. Néanmoins, dans ce qui suit, j'aimerais prendre cette hypothèse comme cadre général. Je procéderai en trois temps.

1. Dans un premier temps, exemples à l'appui, je ferai apparaître concrètement la pulsation sémantique de la traduction.

2. Ensuite, je propose un détour historique en mettant brièvement un corpus historique en parallèle avec les textes modernes afin d'ouvrir un espace interprétatif et d'introduire un paramètre historique. Ceci nous permettra, je l'espère,

3. dans un troisième temps, de mieux comprendre notre propre attitude en face de l'opération traduisante et de nous poser, par ricochet, des questions sur la traduction, et sur l'espace que nous faisons des termes qui s'y rattachent.

Pour le parcours des lieux communs du discours sur la traduction, je me référerai, de manière peu systématique, à des textes qui portent sur la traduction en général. J'ai choisi, un peu au hasard dans le vaste répertoire qui est à notre disposition, des exemples de R. Jakobson, O. Paz, H. Meschonnic, W. Benjamin, et Jacques Derrida et ses interlocuteurs lors d'une table ronde sur la traduction (Montréal 1979, publié dans l'Oreille de l'autre). Autant de textes et d'auteurs qui occupent aujourd'hui une place canonique dans la théorie de la traduction.

Schématiquement parlant, voici comment se présente la pulsation de la traduction dans ces textes et, j'en suis persuadé, dans bien d'autres. C'est un mouvement en trois étapes :

a) il y a d'abord l'extension maximale du terme : la généralité de l'opération traduisante ;

b) ensuite ce champ étendu est découpé, articulé en différentes zones. Des distinctions s'introduisent, des classifications s'opèrent.

c) Finalement, le champ de la traduction se rétrécit et vient à coïncider avec une zone privilégiée, souvent identifée par l'adjectif "véritable" ou " authentique".

Inutile de dire que les manifestations textuelles ne présentent pas tel quel ce schéma qui a le statut d'une reconstruction. Souvent le déroulement syntagmatique et argumentatif présente des inversions, des sauts. des retours en arrière. Souvent les transitions ou les différentes étapes restent implicites. Bien des fois le choix des exemples donnés porte le poids de l'argument. Souvent le non-dit ou l'impensé du texte est aussi important que ce qui est explicite et apparaît dans les arguments développés.

À titre d'exemple - et c'est là un exemple modèle - voici comment se déroule le texte "Traduction : littérature et littéralité " de $O$. Paz (traduit de l'espagnol).

Le premier paragraphe s'ouvre sur une affirmation des plus générales : "Apprendre à parler, c'est apprendre à traduire » (26). Aussitôt, la spécificité d'un parler traduisant est effacée, noyée dans la généralité du parler tout court. Cette cœxtensivité de parler et de traduire est reprise un peu plus tard : " ... le langage lui-même, en son essence, est déjà une traduction " (28). Cette équation entre langage et traduction induit un développement intéressant que je reconstruis comme suit. Puisqu'on est toujours déjà dans le langage, on est également toujours déjà dans la traduction. "Aucun texte n'est entièrement original " (28). Ce qui amène la formule "traduction de traduction de traduction » (28). L'opération traduisante se présente donc sans origine ni fin, ce qui fait sauter 
le face à face inégal de l'original et de sa reproduction, du texte premier et du texte second avec toutes ses implications idéologiques, un peu à l'instar de la formule nietzschéenne de "l'interprétation de l'interprétation de l'interprétation " qui fait sauter les assises d'une certaine herméneutique postulant un sens premier.

Le deuxième pas de $\mathrm{O}$. Paz reste implicite. On peut toutefois le rendre "visible" en opposant la question initiale de l'apprentissage du langage, du parler donc, à l'apparition inopinée du terme texte. À partir d'un moment précis la traduction se trouve être intimement liée à une opération textuelle, perd donc sa généralité absolue. Implicitement, $\mathrm{O}$. Paz distingue une traduction orale et une traduction à partir de textes écrits ${ }^{2}$, pour ne parler d'ailleurs plus que de cette dernière. Ce privilège accordé à la forme écrite de la traduction n'est pas assumé explicitement. Il constitue un des glissements intéressants dans la pulsation sémantique qu'exécute le terme "traduction " sous la plume d'Octavio Paz. Si apprendre à parler, c'est apprendre à traduire, comment se fait-il que, d'un coup, la traduction voit rétrécir son champ aux contours de la textualité écrite ${ }^{3}$ ?

Le prochain glissement ne tarde pas à se produire. Avant le milieu du texte, l'auteur précise en passant, dans une proposition subordonnée : «... la traduction, laquelle est toujours une opération littéraire " (28). Ne nous soucions pas de l'éventuelle contradiction avec la première phrase du texte, ou plutôt de son oubli, et continuons à lire pour trouver quelques lignes plus bas le passage de "littérature » à "poésie "4. Ce dernier terme identifie le lien où $O$. Paz entend situer la question de la traduction. Non seulement ne perd-il plus de vue la question de la poésie jusqu'à la fin du texte, mais il perd presque de vue la question de la traduction. Il est hors de doute que pour $\mathrm{O}$. Paz la zone privilégiée de la traduction est celle où traduction et poésie coïncident et deviennent cœxtensives. Le champ sémantique est arrivé à son extension minimale, sa charge qualitative a atteint son intensité maximale.

On m'objectera qu'un poète comme $\mathrm{O}$. Paz a bien le droit de s'intéresser - même exclusivement - à la spécificité poétique. Deux réponses sont à donner : d'abord je rappelle mon objectif de ne faire, dans un premier temps, que décrire les pulsations sémantiques de la traduction dans certains textes, et de les expliciter là où elles restent implicites. Ensuite, le mouvement décrit dans un texte d'O. Paz n'est pas du tout particulier à O. Paz : il semble constituer la règle d'une certaines théorisation de la traduction.

De la plus grande généralité de la traduction on va tout droit à l'exclusivité de sa forme écrite pour la ramener finalement au cercle étroit du littéraire ou du poétique. On peut d'ores et déjà formuler la question de savoir ce qui vaut à l'écriture et a fortiori à l'écriture poétique ce privilège qui, de surcroît, se présente souvent comme allant de soi.

Avant d'amorcer une réponse observons quelques variantes que nous offrent d'autres textes :

"Aspects linguistiques de la traduction " de Jakobson est probablement aujourd'hui un des textes les plus canoniques sur la traduction. Peu de "traductologues" résistent à la tentation de le citer. Je ne fais pas exception. On sait que Jakobson s'autorise à généraliser l'opération traduisante par un renvoi au " plus profond investigateur de l'essence des signes " (79), Ch. S. Peirce. Il fait aussi coïncider la généralité de la traduction avec celle de la pratique des signes ("semiosis ") : "le sens d'un mot n'est rien d'autre que sa traduction par un autre signe" (79).

Ici, le geste généralisateur est encore plus audacieux que chez $\mathrm{O}$. Paz car il porte la traduction au-delà du domaine linguistique. Une conséquence logique et pratique est à signaler : tout terme dont le domaine d'usage se généralise affaiblit son pouvoir discriminatoire. Si toute pratique de signes devient traduction, alors l'universalité de la traduction efface le pouvoir explicatif et cognitif du terme "traduction", du moins dans un contexte analytique où il fonctionne de manière opératoire. Cela n'empêche pas le mou- 
vement d'expansion d'avoir, en tant que tel, une valeur stratégique et cognitive en tant que déplacement historique d'une problématique ${ }^{5}$. Quand Jakobson découpe par la suite ce champ étendu, où sémiose et traduction sont synonymes, en trois opérations ou zones différentes (traduction intra-, interlinguale, intersémiotique), il identifiera quand même la traduction interlinguale comme la traduction proprement dite. Et on est en droit de se demander — avec J. Derrida dans l'Oreille de l'autre (143) - si Jakobson n'accède pas à son étendue maximale de la traduction par une simple opération métaphorique ${ }^{6}$ à partir du "référent modèle " que serait la traduction interlinguale.

Malgré sa perspective spécifiquement linguistique, Jakobson finit quand même par dériver, à la fin de son texte, vers la question de la traduction poétique - ne fût-ce que pour en constater l'impossibilité. C'est là une manière spéciale de privilégier une zone spécifique de la traducton. Manière qui, par ailleurs, confirme implicitement le privilège accordé à la forme écrite de la traduction, ce qui n'est certainement pas conforme à la généralité de la sémiose peircienne. Si la traduction se trouve réduite à zéro dans l'impossibilité de la traduction poétique (86), la poésie s'en trouve d'autant plus rehaussée.

Dans "Poétique de la traduction » de Henri Meschonnic et dans "La tâche du traducteur " de Walter Benjamin, deux textes complexes dont nous ne pouvons malheureusement traiter que tangentiellement, on constate la collusion implicite entre traduction, textualité, écriture et poésie7. Ces quatre termes fonctionnent dans un circuit de renvois circulaires qui produisent une quasi-synonymie. Pour aller vite, on dirait que le fait saillant commun à ces deux auteurs consiste en une certaine sacralisation de l'écriture, bien que, par ailleurs, leurs textes soient très différents.

Cette identification d'une zone privilégiée va de pair avec une distinction hiérarchique de différentes pratiques de la traduction utilitaire, s'inscrivant dans le contexte pragmatique d'une communication "véhiculaire " (Meschonnic, 345) apparaît comme moins intéressante, moins valorisée que les " traductions qui soient plus que des transmissions " (Benjamin, 60). Benjamin usera d'antinomies sémantiques telles que profondeur/surface, essentiel/inessentiel pour marquer cette hiérarchisation. Finalement, la "véritable traduction" ou la "bonne " théorie de la traduction a partie liée avec une théologie de l'écriture. Elle se situe dans une zone où l'écriture est engagée dans un "processus de sacralisation " (Derrida 1982:195) et donne lieu au texte sacré. Elle s'articule au seuil où l'écriture se transfigure en prenant un $\mathrm{E}$ majuscule.

Cette sacralisation adopte des formes variables. Chez Benjamin elle s'exprime par un messianisme explicite de la traduction qui est alors mouvement vers un langage à venir appelé " langage à l'état pur " ("reine Sprache »). Chez Meschonnic le même élément se manifeste par une omniprésence des termes texte et écriture. Quelque différencié et innovateur que soit par ailleurs le travail de Meschonnic, la fréquence de ces deux termes a un effet pléthorique. Énoncé dans le contexte d'une critique générale de l'idéologie de la transparence, de l'originalité du texte créateur et de la fonction "véhiculaire " du langage, le lien étroit, sinon exclusif entre traduction, texte et écriture finit par inscrire la traduction dans une zone privilégiée. De surcroit, le texte de Meschonnic est écrit d'un ton affirmatif, sinon prescriptif :

Si la traduction d'un texte est texte, elle est l'écriture d'une lecture-écriture. (354)

Traduire est donc dans la pratique et la théorie de l'écrire. (352)

Il faut que la théorie de la traduction soit la théorie d'une pratique du traducteur homologue à l'écrire.

On peut dès maintenant se demander si une telle insistance sur l'écriture et sur le textuel, à l'occasion de la traduction, n'indique pas une attitude culturelle très générale à l'égard des pratiques sémiotiques, attitude qui serait du même ordre que le phonocen- 
trisme que Jacques Derrida nous a fait voir dans notre culture. Il faudrait alors admettre l'existence d'une tendance qu'on pourrait appeler "graphocentrisme " pour marquer, par ce nom, sa symétrie par rapport au " phonocentrisme». Ces deux tendances se manifestent dans les textes de la même manière implicite et indirecte. Elles se situent au même niveau de profondeur de la pratique discursive : avançant sous le couvert de l'évidence, elles deviennent invisibles et véhiculent de l'impensé. Le "graphocentrisme" consisterait, cependant, à donner la priorité aux formes écrites de la pratique des signes et à oublier — pour ne pas dire réprimer - les formes orales. Il n'y a pas de raison autre que la force d'une certaine tradition pour " oublier " systématiquement les formes orales de la traduction dans le discours théorique sur la traduction en général. Il nous faut apprendre à penser cette tradition et, par la suite, soit l'assumer explicitement, soit la critiquer.

Ces deux tendances ont une relation nécessairement conflictuelle. Dans un travail bien plus vaste que ce qu'il est possible d'accomplir sur la base d'un corpus limité d'écrits sur la traduction, il s'agirait de retracer historiquement leurs conflits et peutêtre d'identifier leur filiation culturelle. Comme le travail sur le phonocentrisme a été commencé et avancé de manière convaincante par Jacques Derrida, le moment est venu de se pencher sur le "graphocentrisme ". Cette manière de procéder n'implique d'aucune manière qu'on accorderait à l'une des tendances la préséance sur l'autre.

De nouveau on pourrait formuler des objections : chez Benjamin, par exemple, la forme écrite et poétique de la traduction se trouve être privilégiée pour une raison bien précise. Son texte figure comme préface à une traduction poétique justement. Cela est exact, mais n'empêche pas le texte de Benjamin de prendre une allure très générale (son titre l'indique) et de se détacher de la contingence du contexte, fidèle en ceci à ce que son contenu stipule.

Plutôt que de limiter la validité du texte de Benjamin par son contexte d'énonciation, il me paraît plus important de faire apparaître la manière dont la question de la traduction s'y trouve intimement liée à une certaine théorisation du poétique et de constater que chez Benjamin, les deux problématiques pointent vers le sacré. Le texte sacré figure dans le texte de Benjamin comme un point culminant qui a la fonction d'un lieu asymptotique. Le moment décisif où traduction et poésie prennent cap sur le sacré est très explicitement marqué par l'auteur :

Et, si l'essence ultime qui, là, est pur langage en soi, n'existe dans les langues que liée à des éléments verbaux et à leur variation, il faut bien que, sur les œuvres elles-mêmes, pèse le faix d'un sens venu de l'extérieur. Les délivrer de ce poids, faire du symbolisant le symbolisé même, restaurer le pur langage qui a pris forme dans le mouvement des langues, - tel est, unique et violent, le pouvoir de la traduction. (71)

Si je « traduis » ce qui me paraît décisif ici dans le langage qui nous est bien familier en études littéraires depuis quelques années, c'est du passage d'une référence externe à une référence interne ("faire du symbolisant le symbolisé ") qu'il y va. C'est la théorie de l'autoréférence et de l'autotélisme de la littérature qui est en jeu ici. Et elle restera en jeu par implication tant que nous faisons de Walter Benjamin la figure de proue de la théorie de la traduction. La question pour nous est de savoir si nous voulons l'assumer avec sa trajectoire ouverte sur le texte sacré.

Si j'entame ici le détour historique annoncé, ce n'est pas pour me lancer dans une élucidation philologique et étymologique des termes dont les pulsations nous occupent ; c'est là un travail important, que d'autres ont fait, et bien fait ${ }^{8}$. C'est plutôt dans une visée herméneutique : donner à nos questions la profondeur historique qui permettra de mieux comprendre notre actualité. En ceci, je prends comme modèle la démarche de 
Manfred Frank qui, dans son livre Der kommende Gott (le Dieu à venir), aborde la question de notre (post) modernité par un détour historique.

Et je reculerai, comme lui, de quelque deux cents ans et choisirai le passage du $\mathrm{XVIII}^{\mathrm{e}}$ au XIX ${ }^{\mathrm{e}}$ siècle comme analogon historique de notre situation. Je suis conscient, ce faisant, de m'orienter du côté de ceux qui apparaissent chez Meschonnic - et chez bien d'autres - comme les ennemis idéologiques : les idéalistes. C'est que je ne suis pas d'accord avec la facilité que nous nous donnons en usant de l'étiquette "idéalisme ", " les idéalistes " pour jeter aux oubliettes de l'histoire une pensée et des textes très riches et qui nous sont proches. Il nous faut plutôt les relire, ne fût-ce que pour mieux nous comprendre dans notre différence par rapport à eux. Par exemple par rapport à Schleiermacher qui figure ici comme témoin principal. Il est l'auteur d'un texte sur la traduction qui est certainement, entre autres, d'inspiration idéaliste (surtout par la théorie du sujet qu'il comporte). Il s'agit d'un discours sur les méthodes de la traduction, que Schleiermacher a lu en 1813 à l'Académie royale de Berlin'.

Ce texte représente encore aujourd'hui une des contributions les plus intéressantes sur la question de la traduction. C'est que, dans ses trente pages, il expose cette question dans un contexte extrêmement vaste, faisant apparaitre les imbrications de l'opération traduisante avec l'essor du commerce international ("Geschäftsleben»10), avec les relations entre États, avec la compréhension en général et celle qu'un individu peut avoir de lui-même, avec l'histoire et le développement des langues, avec l'élaboration d'une pensée nationaliste et avec le développement de la production culturelle. Certes, on ne saurait analyser ici toute la complexité de ce texte, mais même en procédant de manière sélective, on pourra faire ressortir quelques contrastes qui donneront du relief aux textes plus récents que nous venons d'examiner. Chez Schleiermacher, bien qu'il y ait une hiérarchisation analogue de différentes opérations traduisantes, il n'y a pas pour autant de rejet de la traduction utilitaire. Au contraire, il reconnaît l'intérêt pratique pour l'État, la société et le commerce de ce qui constitue quantitativement la plus grande activité traduisante et il sait l'articuler, en l'intégrant dans une réflexion plus vaste, avec l'aspect plus ésotérique et plus élitaire qui constitue, chez lui aussi, la zone privilégiée de la traduction, à savoir la traduction philosophico-poétique.

Voici comment Schleiermacher articule les différentes opérations traduisantes.

\begin{tabular}{|c|c|c|c|}
\hline \multirow[b]{3}{*}{ Domaine } & \multicolumn{3}{|c|}{ Traduction (Uebertragen, 42) } \\
\hline & $\begin{array}{l}\text { Traduction pratique } \\
\text { (Dolmetschen) }\end{array}$ & \multicolumn{2}{|c|}{$\begin{array}{l}\text { Traduction proprement dite } \\
\text { (eigentliches Uebersetzen) }\end{array}$} \\
\hline & $\begin{array}{l}\text { Échanges pratiques } \\
\text { (Bürgerlicher } \\
\text { Verkehr, 42) }\end{array}$ & & Art \\
\hline $\begin{array}{l}\text { Mode de } \\
\text { communication }\end{array}$ & Oral & \multicolumn{2}{|c|}{ Écrit } \\
\hline $\begin{array}{l}\text { Spécificités } \\
\text { sémiotiques }\end{array}$ & $\begin{array}{l}\text { Pratique des signes centrée sur } \\
\text { la référence comme élément } \\
\text { stabilisateur }\end{array}$ & \multicolumn{2}{|c|}{$\begin{array}{l}\text { Pratique des signes centrée sur } \\
\text { l'acte discursif individuel comme } \\
\text { élément créateur }\end{array}$} \\
\hline Fonction & $\begin{array}{l}\text { Répétition mécanique } \\
\text { reproduction }\end{array}$ & \multicolumn{2}{|c|}{$\begin{array}{l}\text { Transformation } \\
\text { production-création }\end{array}$} \\
\hline
\end{tabular}


L'intérêt du texte de Schleiermacher réside donc d'abord dans le fait qu'il articule de manière compréhensive toutes les activités traduisantes qui ont cours dans la société moderne. J'insisterai sur ce point en m'appuyant sur une des métaphores du texte de Benjamin :

Pour qu'on puisse reconstituer la totalité d'une amphore à partir de ses débris, il faut que chacun d'eux, dans sa plus infime singularité, s'adapte à son voisin plutôt qu'il ne s'y apparie. De la même façon, au lieu de s'identifier au sens du texte, la traduction doit bien plutôt, par un mouvement d'amour qui s'étend au détail, incorporer dans sa propre langue le mode de visée qui était celui de l'original ; ainsi, comme les tessons s'avèrent morceaux d'une même amphore, les deux textes se révèlent fragments d'un seul langage. (69)

Cette "métaphore ", comme l'appelle Jacques Derrida (1982 : 162) figure chez Benjamin la visée transcendante de la traduction et a l'avantage d'articuler cette visée dans l'ambivalence de sa dimension à la fois nostalgique $^{11}$ et utopique (messianique). Un contemporain de Schleiermacher, Kleist a exploité le même potentiel de figuration dans sa pièce de théâtre la Cruche cassée. Cependant, à l'instar de Schleiermacher et contrairement à Benjamin, Kleist a articulé en même temps que le sens transcendant, un sens pratique de la même métaphore. D'une part Dame Marthe, la propriétaire de la cruche, cherche à obtenir du tribunal l'identification de celui qui l'a cassée et, par conséquent, des réparations matérielles. D'autre part, sa quête juridique est continuellement projetée sur un plan moral et herméneutique à résonnance métaphysique, où il y va de l'intégrité perdue du sens et de l'innocence. L'intérêt de la pièce se trouve dans l'imbrication de ces deux niveaux du jeu dramatique. Chez Kleist et Schleiermacher la dimension pratique et utilitaire n'est pas une contrainte dont il faut se libérer pour accéder au langage à l'état pur, mais c'est dans et avec la pratique de la communication quotidienne, enracinée dans les réalités historiques, économiques, politiques, que s'articulent les questions théoriques.

Après la lecture de Paz, Jakobson et Meschonnic, celle de Schleiermacher produit un effet presque " unheimlich " de familiarité : le schéma que j'ai élaboré à partir des textes plus récents y est déjà entièrement à l'œuvre. D'une généralité maximale du traduire on passe à une classification de différentes opérations traduisantes, pour s'arrêter longuement et définitivement sur une seule parmi celles-ci : la traduction proprement dite (" eigentlich ", 39) qu'est, chez Schleiermacher aussi, la traduction de l'œuvre d'art poétique.

Ce qui est différent chez Schleiermacher cependant, c'est la manière dont il met ce schéma en texte : tous les mouvements, toutes les pulsations sémantiques de la traduction sont assumés chez lui par une augmentation explicite. Surtout aussi, chose rare ou inexistante dans les textes plus modernes, le rétrécissement du champ généralisé de la traduction à une zone privilégiée. S'il donne la préférence à la traduction littéraire (le geste n'est pas moins innocent, moins chargé de présuppositions que chez les auteurs modernes), toutefois, il n'oublie pas ni ne rejette tout le vaste secteur d'une autre activité traduisante dont il fait ressortir l'importance sans pour autant lui accorder un traitement égal. Il appelle ce secteur «Dolmetschen » et l'oppose à celui de «Übersetzen». Le terme "Dolmetschen" couvre un champ plus vaste que ce qu'on désigne aujourd'hui par " interprétariat " en français, car il n'exclut pas la réalisation écrite de l'opération traduisante.

Son choix de traiter de la traduction littéraire en priorité est motivé, et ce sur une base idéologique que nous avons appris à identifier comme "idéaliste »: il attribue à l'acte de parole ${ }^{12}$ qui émane de la subjectivité individuelle du créateur-poète le pouvoir de changer, même de violenter, le patrimoine collectif que représente le système ou la structure de la langue et de contribuer de la sorte et d'une manière décisive au façonne- 
ment de la langue maternelle. $\grave{A}$ plus forte raison, la traduction des grandes œuvres étrangères contribue à l'enrichissement d'une langue pour peu que celle-ci soit capable de recevoir la marque ou l'infléchissement de l'altérité. En ceci il distingue le français de l'allemand. Le français a atteint l'âge mûr ou la rigidité classique et ne peut donc plus bénéficier de l'apport des traducteurs. L'allemand est moins évolué autour de 1800 et, par conséquent, prêt à s'élargir sous l'influence d'une massive activité de traductions philosophiques et littéraires. Un regard empirique sur l'histoire de la traduction peut confirmer ce qui était alors une manière hautement spéculative de voir les choses. La comparaison des adaptations françaises de Shakespeare par exemple avec les monuments de traduction ${ }^{13}$ qu'ont apportés l'idéalisme et le romantisme à la culture allemande est éloquente.

Mais là n'est pas notre question : celle-ci se concentre en ce moment sur les évidences d'une grande ressemblance de "notre" théorie de la traduction avec celle des idéalistes/romantiques et sur l'obligation qui en découle pour nous d'assumer aussi explicitement qu'eux le socle des présuppositions qui la porte.

En élargissant la problématique, on pourrait aussi se demander si les situations historiques, ayant produit ces remarquables ressemblances dans les textes, présentent elles-mêmes des ressemblances. L'intérêt massif autour de 1800 pour pratique et théorie de la traduction et plus particulièrement pour la traduction-transformation et pour la traduction-production serait-il le symptôme d'une crise analogue dans les institutions de la pratique des signes? La question doit rester ouverte, pour nous donner l'occasion de nous tourner encore une fois vers le poétique. Après Albert Béguin et Maurice Blanchot ce sont Philippe Lacoue-Labarthe et Jean-Luc Nancy qui ont rendu accessible au monde francophone la théorisation performative du poétique chez les premiers romantiques allemands.

Comment ce poétique s'articule-t-il avec la traduction chez Novalis? Dans le fragment des Pollen $n^{\circ} 68$, un des plus longs de ce recueil, Novalis esquisse une vaste théorie de la traduction qui, elle aussi, reproduit le schéma tripartite qui nous est devenu familier. Cependant, l'articulation du texte novalisien, moins facile à retracer du fait de sa nature fragmentaire, semble renverser la direction du mouvement argumentatif et ne s'ouvrir sur la généralité maximale qu'à la fin. Novalis commence par distinguer trois types de traductions et il les identifie par les trois adjectifs allemands "grammatisch", "verändernd", " mythisch". La traduction grammaticale produit des traductions au sens commun ${ }^{14}$ du terme. Elle implique beaucoup d'érudition et ne demande que des capacités " discursives ". À ceci s'opposent les " capacités poétiques 》 ("poetischer Geist ") requises par les deux autres types de traduction : la traduction transformante (angl. transformative, 69) a partie liée avec parodie et pastiche (le terme allemand est " Travestie ". Elle comporte une potentiation de l'acte poétique, le traducteur y devient "le poète du poète " et doit être capable d'articuler en même temps le même et l'autre, son idée à lui et celle du poète qui est un autre, mais aussi l'idée propre au poète et l'altérité qu'il y apporte en tant que traducteur. Le résultat est une transformation, ou pour le dire dans une figure chère à Goethe quand il parle de la traduction, l'établissement d'un troisième lieu ${ }^{15}$ qui aura déplacé et le même et l'autre.

Finalement, la traduction mythique demande la pénétration des esprits poétique et philosophique. Son résultat dépasse le domaine langagier. Novalis donne deux exemples :

La mythologie grecque est en partie issue d'une telle traduction d'une religion nationale. La figure moderne de la sainte vierge est également un mythe de ce type. (II, 438)

Ici nous retrouvons l'articulation du poétique et du philosophique sur le sacré, ce qui nous " rappelle " certaines implications du texte de Benjamin. De Benjamin à Novalis et 
aux romantiques plus généralement il y a en fait des liens importants dont il faut faire mention, même s'il est impossible de les examiner en profondeur ici. D'abord, ils ont en commun non seulement un intérêt particulier pour le religieux, mais aussi, dans les deux textes que nous considérons ici, une manière indirecte d'inscrire cet intérêt capital dans le texte. Chez Novalis, le religieux entre par la porte des exemples, chez Benjamin il y a une thématisation très explicite et répandue du sacré, et par moments des réflexions sur la fonction positives des religions ${ }^{16}$, sans parler du messianisme qui soustend toute sa réflexion sur la traduction.

Ensuite, Benjamin rapproche lui-même son concept de "survie de l'œuvre »17 des concepts d'ironie et de critique d'art romantique. Et il est bien placé pour le faire étant donné que sa thèse de doctorat portait sur le concept de la critique d'art à l'époque romiantique. Les opérations de la traduction, de l'ironisation (au sens non tropologique, philosophique du premier romantisme allemand) et de la critique auraient donc toutes pour effet d'effectuer la survie de l'œuvre en déployant un potentiel contenu en elle et en le projetant au-delà des contingences de sa première réalisation. C'est dans ce sens qu'on peut lire la description novalisienne des traductions mythiques :

Elles représentent le caractère pur et accompli de l'œuvre d'art individuelle. Elles ne nous donnent pas l'œuvre d'art réelle, mais son idéal. (II, 438)

A la fin du fragment, Novalis ajoute :

Non seulement des livres, mais tout peut être traduit d'après ces trois manières. (II, 440)

Il projette de la sorte l'opération traduisante sur un horizon de généralité illimitée. Les implications logiques du texte veulent que cet horizon coïncide avec celui de l'opération poétique, du moins dans les deux types de traduction impliquant un "esprit poétique ". Encore une fois, poésie et traduction viennent à coïncider dans leur extension, mais ce qui est nouveau, c'est que cette ccextensivité ne se produit pas dans une zone limitée, au contraire, elle se présente dans la généralité maximale des deux termes. Chez Novalis, ainsi que chez ses amis d'Iéna, la poésie suit la traduction - ou vice versa - dans son amplitude sémantique maximale. Le poétique est sorti de son ghetto esthétique, il quitte sa marginalité, il se déplace vers le centre, c'est-à-dire qu'il est partout, principe général de productivité discursive tout court. Car, comme le roman est appelé à devenir le genre unique, englobant en les mélangeant tous les genres littéraires, ainsi la poésie est appelée à devenir la discursivité tout court. En tant que dénominateur commun de tous les systèmes des discours, la poésie vient à épouser les contours de la traduction qui, bien qu'articulée de manière idéaliste sur la base d'un concept de création originale et d'individualité de l'œuvre, devient également principe de production. Dans l'univers poétique, tout devient en principe traduisible en tout. L'opération traduisante devient le garant pratique en même temps que le principe d'expansion illimitée de l'universalité poétique.

On reconnaît ici des traits importants de notre théorisation moderne de la traduction : le même intérêt pour la mobilité sémiotique, pour le potentiel transformateur et producteur de l'opération traduisante. Mais il y a lieu de se demander où nous en sommes dans notre théorisation du littéraire, du poétique qui est si intimement lié avec la question de la traduction. La base idéologique idéaliste n'étant plus viable, sur quelle base pensons-nous l'activité poétique ? Quelle extension sommes-nous prêts à lui donner? Ces questions ne suggèrent point la construction d'un autre parmi les "méta-récits" dont a parlé Lyotard récemment. Mais elles devraient nous provoquer dans la mesure où nous ne sommes peut-être pas aussi " avancés " que nos prédécesseurs idéalistes sur ce point. Elles nous provoquent à dire ce que nous tenons d'eux et ce qui fait la différence historique de notre position par rapport à eux. 
Notre théorie de la littérature ne me paraît pas si clairement articulée, surtout quand elle s'inscrit en creux dans les éléments restés implicites, dans les ellipses d'argumentation d'une théorie de la traduction qui accorde au littéraire une place privilégiée.

Ou encore dans les exemples, selon le principe formulé par Meschonnic : "toute théorie tient plus qu'elle ne croit tenir, à ses exemples " (Pour la poétique, II, 335). Dans le texte publié de la table ronde déjà mentionnée sur la traduction, Jacques Derrida commence sa première réponse par : "je vais prendre deux exemples pour commencer " (132). Il s'agit de deux exemples littéraires : Finnegans Wake de Joyce et "Pierre Ménard auteur du Quichotte » de Borges. Ce premier geste, recours immédiat à la littérature pour exemplifier la traduction, dans un contexte où l'on parle de la traduction au sens le plus général, ce geste est significatif à plusieurs égards. D'abord il confirme, en s'y inscrivant, la tradition dont nous venons d'esquisser à la fois l'actualité et la profondeur historique. Ensuite, il identifie d'emblée un lieu privilégié de la réflexion sur la traduction.

Si le choix d'exemples littéraires est en soi significatif, celui des deux auteurs en particulier l'est à plus forte raison. Il véhicule une idée très spécifique de la littérature. Joyce et Borges jouissent aujourd'hui d'une grande popularité auprès d'un public peu populaire. Ce sont des auteurs à la mode parmi les initiés de la chose littéraire. Car c'est sur eux que se projettent les idées des théoriciens littéraires : Joyce les intéresse pour son exploitation infinie du signifiant, pour sa prolifération de la signifiance dans le langage. Borges pour sa mise en abîme thématique et narrative du processus de représentation qui, pris dans de multiples réflexions spéculaires, s'en trouve être sans fond, sans référence, sans origine, sans ancrage ontologique. Plus que d'autres, ces deux auteurs se prêtent à la célébration d'une prolifération du langage à l'infini, du lestage de toute référence externe, à l'oubli des fonctions pratiques et idéologiques de la littérature. Les exemples choisis suggèrent donc une littérature, et sa théorie, basée sur les principes de l'autogénération et de l'autoréférence du texte écrit. Une écriture littéraire qui, affranchie des contraintes pratiques, se produirait à l'état pur. La théorie littéraire qu'on peut de la sorte extraire des exemples auxquels elle tient nous ramène à Walter Benjamin.

Or, c'est justement le texte de Benjamin sur la traduction que Jacques Derrida rejoint à partir des exemples littéraires, et ce après un passage par le texte sacré de l'Écriture. Et Benjamin deviendra l'exemple paradigmatique du théoricien sur la traduction. Jacques Derrida, guidé par ses interlocuteurs, touchera à d'autres exemples et noms : Freud, Lacan, Heidegger, Hegel, Blanchot, Jakobson, mais sa propre réflexion sur la traduction ramène toujours à nouveau son exemple à lui, Benjamin.

Benjamin est le théoricien récent de la traduction qui relie le plus explicitement le travail du "véritable traducteur » $(65)$ - celui qui est affranchi de l'obligation pratique de communiquer un sens - le poétique et le sacré dans une tradition qui se concentre sur l'écriture. Ce lien importe beaucoup à Jacques Derrida. Adopte-t-il en ceci la position de Benjamin? Il y a des moments où, par un effet de glissement, tel semble être le cas. Qui parle par exemple ici : « faire du poétique, c'est faire du sacré, en ce sens-là c'est faire de l'intraduisible " (196) ? Pourtant Jacques Derrida, répondant à une question sur le sacré, prend ses distances par rapport à ce qu'il y a de gênant chez Benjamin (194), à savoir son insistance sur le caractère messianique de la traduction. Il se dissocie donc des implications religieuses de son exemple. Il fait un effort minutieux pour séparer le sacré et le religieux, pour tirer le sacré du côté du poétique. Mais aussi le poétique du côté du sacré. Cette tendance à la sacralisation de la parole écrite-poétique ne réintroduit-elle pas une forme de religiosité très spéciale : une religion de l'écriture poétique ? Retour du religieux d'autant plus plausible qu'il est confirmé par la dénégation de la dimension religieuse dans le texte de Benjamin. 
Si cette tendance se confirmait, deux tâches deviendraient urgentes. D'une part soumettre à une critique radicale l'acceptation implicite d'une zone privilégiée de la traduction résultant de la superposition de traduction et poésie. D’autre part, et en conséquence, dissocier littérature et traduction pour penser chacune de ces pratiques discursives en son lieu propre. Cela permettrait, d'un côté de reprendre la théorie de la traduction dans toute son amplitude esquissée par Schleiermacher, incluant donc la traduction orale, et de l'autre côté de reprendre la réflexion sur le littéraire sans l'engager d'emblée et exclusivement sur la voie étroite de la sacralisation de l'écriture.

\section{Notes}

1. La notion texte a suivi un double mouvement analogue. En plus de son expression généralisante, elle a subi un changement de sens : d'objet d'analyse matériellement et objectivement donné, le texte s'est mué en principe de productivité sémiotique, de produit en processus.

2. Je décide ici, un peu arbitrairement, que le texte implique une réalisation par écrit. Ceci n`est pas si arbitraire cependant, si on voit comment les textes analysés associent systématiquement écriture et textualité.

3. Cette transformation implicite rappelle cette autre chez Freud où, dans l'évolution de l'individu, on passe de "Kind" à "Knabe", "Jüngling" pour aboutir à "Mann".

4. Ce passage, ainsi que les deux termes impliqués, mériterait un commentaire tout aussi long que l'est cet article sur la traduction. Pour les pulsations de la littérature au cours de son histoire, on se rapportera à Escarpit. Et observons que, dans les textes sur la traduction, la relation entre "littérature " et "poésie " varie également : tantôt les deux termes sont quasi synonymes, tantôt la poésie est une zone privilégiée de la littérature.

5. À titre de renvoi, deux exemples analogues dans des domaines connexes : 1) Aujourd'hui, la généralisation stratégique du terme "fiction " pour questionner la prétention de vérité (référentielle) de certains discours non littéraires. Le "cri de guerre" est alors : tout n'est que fiction. 2) À lépoque romantique, la généralisation du genre romanesque comme genre unique, comme " poème-mélange " ("Mischgedicht ") qui inclurait tous les autres genres. D'où la définition à allure tautologique de $F$. Schlegel : le roman est un livre romantique. Mais cela oblige Jakobson, qui tient à la valeur explicative de ses termes, à procéder à un découpage terminologique et typologique du domaine de la traduction en $\mathrm{y}$ introduisant des souscatégories.

6. Cette métaphorisation de la traduction - une sorte de mise en abîme du mouvement qui " porte de l'autre côté" - mériterait une attention particulière : ne faudrait-il pas aborder l'usage que fait par exemple Michel Serres du terme de traduction sous cet angle de vue?

7. Si le terme " poésie " n'est pas toujours présent explicitement, il l'est souvent implicitement par le choix des exemples.

8. Par exemple Jean Pépin pour hermeneia et G. Folena pour volgarizzare et tradurre.

9. Le titre allemand est "Ueber die verschiedenen Methoden des Uebersezens".

10. "Geschäftsleben" se réfêre cependant plus généralement à tous les aspects pratiques de la vic en société ("Gebiet des bürgerlichen Lebens", comme dira le texte plus loin, p. 42) et non pas exclusivement au domaine économique.

11. Le terme "restaurer" (71, en allemand "zurückgewinnen") actualise cette dimension dans le texte.

12. Le terme allemand est "Rede " qui correspond plutôt à " discours " (oral et écrit) qu'à " texte " en français et n'implique donc, en tant que tel, aucune préséance accordée à l'oral ou à l'écrit.

13. Dans une énumération incomplète, ne mentionnons que l'Odyssée de J.H. Voss, le Shakespeare de A.W. Schlegel, le Platon de Schleiermacher, le Don Quichotte de L. Tieck. Rappelons également que Benjamin parle de "l'œuvre considérable de traducteurs" (66) des romantiques.

14. Novalis dit "im gewöhnlichen Sinn ", il évite le terme "eigentlich" (proprement dit) qu'utilisent d'autres théoriciens de la traduction.

15. Cf. Störig, p. 36.

16. Voir à titre d'exemple ce passage :

[...] " toute traduction n'est, de façon ou d'autre, qu'une manière provisoire de se mesurer à ce que les langues ont d'étranger. Venir à bout de cette différence fondamentale sur un mode qui ne serait ni passager ni provisoire, la surmonter d'un coup et pour toujours, c'est là une tâche inaccessible à l'homme ou du moins qu'il ne saurait s'assigner de façon immédiate, ses seules médiatrices étant ici les religions dont le développement peut faire mûrir au sein des langues le germe mystérieux d'un langage supérieur. "(64-65).

17. En allemand il y a deux termes : Fortleben et Überleben. Cf. les remarques de Jacques Derrida à ce sujet dans l'Oreille de l'autre, p. 161. 


\section{BIBLIOGRAPHIE}

BENJAMIN, Walter (1959) : "La tâche du traducteur", in OEuvres choisies, Paris, Julliard, pp. 57-74. DERRIDA, Jacques (1967) : De la grammatologie, Paris, Éditions de Minuit.

ESCARPIT, Robert (1970) : "La définition du terme 'littérature" ", in le Littéraire et le social, Paris, Flammarion, pp. 259-272.

FOLENA, Gianfranco (1973) : " 'Volgarizzare' et ' tradurre' : idea e terminologia della traduzione dal Medio Evo italiano e romanzo all'umanesimo europeo ", in la Traduzione, Saggi et Studi, Trieste, Edizione Lint, pp. $57-120$.

FRANK, Manfred (1982) : Der kommende Gott, Vorlesungen über die Neue Mythologie, Frankfurt, Suhrkamp.

JAKOBSON, Roman (1963) : "Aspects linguistiques de la traduction ", in Essais de linguistique générale, vol. 1, Paris, Éditions de Minuit, pp. 78-86.

LÉVESQUE, Claude et Christie MCDONALD (dir.) (1982) : l'Oreille de l'autre. Otobiographies, transferts, traductions. Textes et débats avec Jacques Derrida, Montréal, VLB éditeur.

MESCHONNIC, Henri (1973) : "Poétique de la traduction", in Pour la Poétique II, Paris, Gallimard, pp. 305-454.

NOVALIS (1960) : Hymns to the Night and Other Selected Writings, trad. Charles E. Passage, Indianapolis, New York, Bobbs-Merrill.

NOVALIS (1981) : Schriften, vol. II, Das philosophische Werk I, Stuttgart, Kohlhammer.

PAZ, Octavio (août 1971) : "Traduction : littérature et littéralité ", Nouvelle revue française, 224, pp. 26-37.

PÉPIN, Jean (1975) : "L'herméneutique ancienne ", Poétique, 23, pp. 291-300.

SCHLEIERMACHER, Friedrich (1983) : "Über die verschiedenen Methoden des Übersetzens ", in Störig, ed., Das Problem des Übersetzens, pp. 38-70.

STÖRIG, Hans Joachim, ed. (1973) : Das Problem des Übersetzens, Darmstadt, Wissenschaftliche Buchgesellschaft. 